\title{
Reversible Logic gate based on QSD Addition/Subtraction using DPG Gate
}

\author{
Asiya Hasan \\ M. Tech. Scholar \\ Dept.of Elecronics and \\ Communication \\ TRUBA, Bhopal, India
}

\author{
Nishi Pandey \\ Assistant Professor \\ Dept.of Elecronics and \\ Communication \\ TRUBA, Bhopal, India
}

\author{
Meha Shrivastava \\ Head of Dept. \\ Dept.of Elecronics and \\ Communication \\ TRUBA, Bhopal, India
}

\begin{abstract}
Arithmetic Logic Unit plays a vital role in the central processing unit of the computer system. Addition is considered to be a primary part in the ALU. Power and speed are the major parameters to be kept in mind for designing an adder. Because of carry propagation, complexity and delay gets introduced in the adder circuit due to which addition, subtraction and multiplication obtains delay in the Arithmetic Logic unit. In order to reduce the delay, carry-free addition is introduced by QSD (Quaternary Signed Digit) Numbers. In this paper, a fast QSD Addition and Subtraction circuit is designed by use of DPG Reversible Logic Gates.
\end{abstract}

\section{Keywords}

Quaternary Signed Digit (QSD), Reversible Gate, DPG Gate, Carry Free Addition

\section{INTRODUCTION}

In digital electronics, computers, arithmetic operations and signal processors play an important and significant role. It means that as soon as the addition and subtraction take place, the speed of the whole system increases. Standard binary systems can produce carry which spreads from the least significant digit to the most significant digit. Thus, the length of the binary number determines the time that is needed for addition. We are introducing the high-speed QSD arithmetic logic unit, which is capable of adding without carry and subtracting without borrow. The QSD addition/subtraction operation uses a fixed number of min terms for any size. The computation time is reduced because in QSD number system, carry propagation chain is eliminated so that the speed of the machine is enhanced. Carry free addition is possible because of the Quaternary signed digit number system. QSD Adder/QSD Subtractor circuits are logical circuits that are designed to carry out arithmetic operations at high speed. The first step is the generation of intermediate carry and intermediate sum between the augend and addend. In the second step the intermediate sum of the current digit iscombined with the carry of the lower significant digit, whichenhances the speed of the machine [1]. The advancement in VLSI designs, portable device technologies and increasingly high computation requirements, lead to the circuit design of faster, smaller and more complex electronic systems at the expense of lots of heat dissipation which would reduce the life of the circuit. Thus power consumption becomes an important issue in modern design [2]. The power dissipation that is tolerable in a given application context is always limited by some practical consideration, such as a requirement that a limited supply of available energy (such as in a battery) not be used up within a given time, or by the limited rate of heat removal in one's cooling system, or by a limited operating budget available for buying energy. Thus, improving system performance generally requires increasing the average energy efficiency of useful operations. It has been unmistakably shown by Frank [3] that reversible figuring is the main feasible alternative to beat the force dispersal [4]. The essential inspiration for reversible registering lies in the way that it gives the main way (that is, the main way that is sensibly steady with the most immovably settled standards of central material science) that execution on most applications inside practical force imperatives may at present keep expanding uncertainly. Reversible logic is also a core part of the quantum circuit model.

\section{LITERATURE SURVEY}

Simranjeet Singh Sudan et al. [1], arithmetic Logic Unit plays a vital role in the central processing unit of the computer system. Addition is considered to be a primary part in the ALU. Power and speed are the major parameters to be kept in mind for designing an adder. In order to reduce the delay, carry-free addition is introduced by QSD (Quaternary Signed Digit) Numbers. In this paper, a fast QSD Addition and Subtraction circuit is designed by use of MIG and COG Reversible Logic Gates.

Radhika Thakur et al. [2], this article is concerned with the construction of a quantum-mechanical Hamiltonian describing a computer. This Hamiltonian produces a dynamical development which emulates an arrangement of basic coherent strides. This can be accomplished if each legitimate stride is locally reversible (worldwide reversibility is lacking). Computational blunders because of clamor can be remedied by method for excess.

Purva Agarwal et al. [3] "review on Reversible common sense Gates and their Implementation", on this paper the Reversible common sense is one of the maximum crucial issue at gift time and it has unique areas for its utility, the ones are low power CMOS, quantum computing, nanotechnology, cryptography, optical computing, DNA computing, virtual sign processing (DSP), quantum dot mobile automata, conversation, laptop photographs. It is impractical to acknowledge quantum processing without usage of reversible rationale. The fundamental motivations behind outlining reversible rationale are to abatement quantum cost, profundity of the circuits and the quantity of trash yields.

Ameya N. Bankar et al.[4], "Design of a novel reversible ALU using an enhanced carry look- ahead adder" reversible rationale is increasing critical thought as the potential rationale outline style for usage in advanced nanotechnology and quantum registering with negligible effect on physical entropy. Late advances in reversible rationale permit plans for PC structures utilizing enhanced quantum PC calculations. Critical commitments have been made in the writing towards the configuration of reversible rationale door structures and 
math units, be that as it may, there are relatively few endeavors coordinated towards.

Tanay Chattopadhyay et al. [5] "layout of green Reversible Binary Subtractors based on a new Reversible Gate", this paper tells approximately the tremendous applications of Reversible common sense in quantum computing, low electricity VLSI design, quantum dot mobile automata and optical computing. While a few scientists have examined the configuration of reversible rationale components, there is very little work provided details regarding reversible paired subtractors. In this paper, we propose the outline of another reversible entryway called TR gate.

\section{PROBLEM STATEMENT}

Moore law states that, the power required for processing just doubles in every 18 months. Irreversible technologies will dissipate a lot of heat and can reduce the life of the circuit. The reversible logic operations do not erase information and dissipate very less heat. As the technology shirking, tradeoff between performance and delay is very essential. The significant interest in delay reduction is consideration of highperformance circuits. Existing design minimizes the delay but at the cost of design metrics. The goal of this thesis is to design various components of a processor with tightly constraints design parameters, which are advantageous than the existing techniques. Reversible logic is a very prospective approach of logic synthesis for delay reduction in future computing technologies.

\section{REVERSIBLE GATE}

Reversible rationale is picking up significance in zones of CMOS configuration on account of its low power dissemination. The customary entryways like AND, OR, XOR are all irreversible doors. Consider the instance of conventional AND entryway. It comprises of two inputs and one yield. Thus, one piece is lost every time a calculation is completed Hence it is impractical to decide a remarkable information that brought about the yield zero. With a specific end goal to make an entryway reversible extra information and yield lines are added so that a coordinated mapping exists between the info and yield. This keeps the loss of data that is fundamental driver of force dispersal in irreversible circuits. The information that is added to a $\mathrm{m} \mathrm{x} \mathrm{n}$ capacity to make it reversible is known as steady information (CI). Every one of the yields of a reversible circuit need not be utilized as a part of the circuit. Those yields that are not utilized as a part of the circuit is called as junk yield (GO). The quantity of trash yield for a specific reversible door is not altered.

The two main constraints of reversible logic circuit is

$>$ Fan out not allowed

$>$ Feedbacks or loops not allowed.

Several $4 \times 4$ gates have been described in the literature targeting low cost and delay which may be implemented in a programmable manner to produce a high number of logical calculations. The DPG gate produces the following logical output calculations:

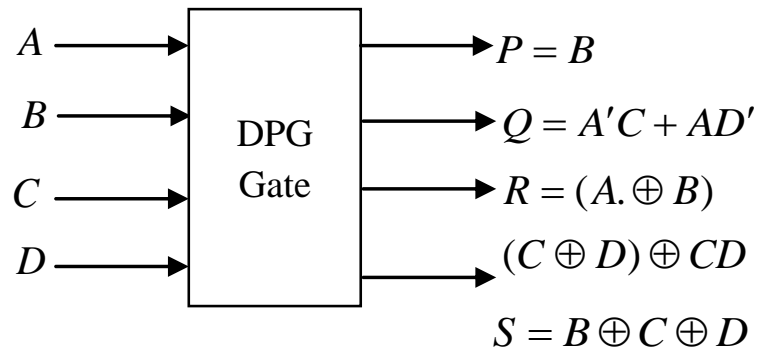

Figure 1: DPG Gate

$$
\begin{gathered}
P=B \\
Q=A^{\prime} C+A D^{\prime} \\
R=(A . \oplus B)(C \oplus D) \oplus C D \\
S=B \oplus C \oplus D
\end{gathered}
$$

\section{PROPOSED DESIGN}

Design to QSD addition and sub-tractor with the help of different types of reversible gate. Low power consumption and low gate count is the main advantage of reversible gate. MIG and COG reversible gate are used to design QSD addition and sub-tractor but more power consumption in existing design.

\section{QSD NUMBER}

1-digit QSD can be represented by one 3-bit binary equivalent as follows:

$$
\begin{aligned}
-3 & =101 \\
-2 & =110 \\
-1 & =111 \\
0 & =000 \\
1 & =001 \\
2 & =010 \\
3 & =011
\end{aligned}
$$

So to convert $n$-bit binary data to its equivalent $q$-digit QSD data, we have to convert this $n$-bit binary data into $3 q$-bit binary data. To achieve the target, we have to split the 3rd, 5th, 7th bit.... i.e. odd bit (from the LSB to MSB) into two portions. But we cannot split the MSB. If the odd bit is 1 then, it is split into $1 \& 0$ and if it is 0 then, it is split into $0 \& 0$. An example makes it clear, the splitting technique of a binary number $(1101101)_{2}$ is shown below: 


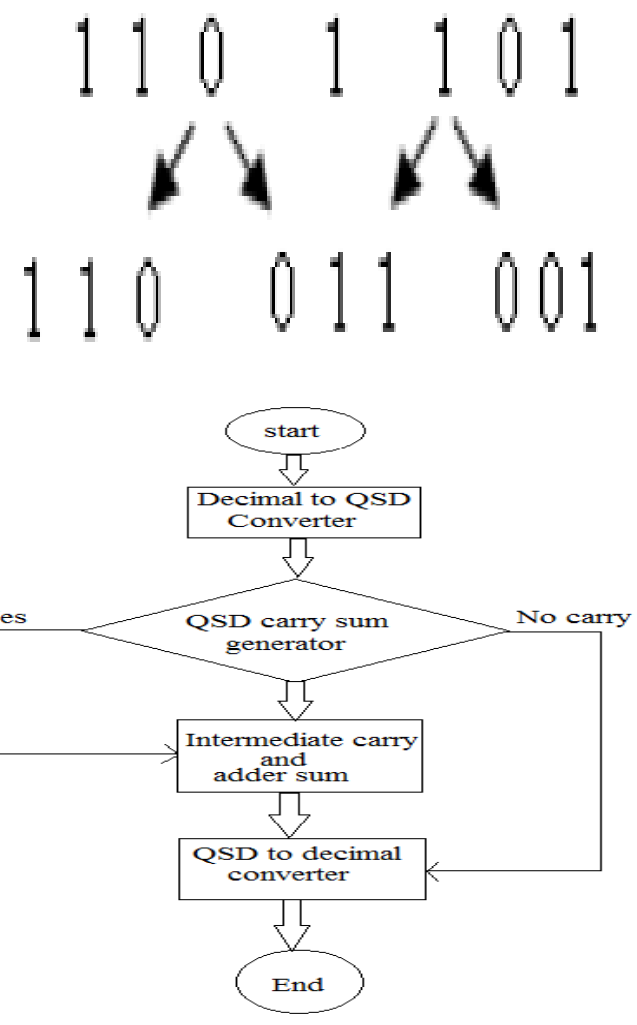

Figure 2: Flow Chart of Proposed Methodology

So we have to split the binary data (1) $q$ - times (as example, for conversion of 2-bit quaternary number, the splitting is 1 time; for converting 3-digit quaternary number the split is 2times and so on). In each such splitting one extra bit is generated. So, the required binary bits for conversion to it's QSD equivalent $(n)=$ (Total numbers of bits generated after divisions) - (extra bit generated due to splitting).

Table 1: Binary representation of Quaternary signed digit numbers

\begin{tabular}{|c|c|c|}
\hline Serial Number & $\begin{array}{c}\text { Quaternary signed } \\
\text { digit }\end{array}$ & Binary Representation \\
\hline 1 & -3 & 101 \\
\hline 2 & -2 & 110 \\
\hline 3 & -1 & 111 \\
\hline 4 & 0 & 000 \\
\hline 5 & +1 & 001 \\
\hline 6 & +2 & 010 \\
\hline 7 & +3 & 011 \\
\hline
\end{tabular}

\section{EVALUATION PARAMETERS}

\section{Garbage Output}

Unused output or extra output is not used to circuit is called garbage output.Garbage output will be decreased.

\section{Constant Input}

The constant inputs either ' 0 ' or ' 1 ' defined in the input are known as ancilla input or constant input.Constant input will be reduced.

\section{Number 4-input LUTs}

LUT stands for look up table that reduces the complex mathematics calculations and provide the reduced processing time. LUT will be reduced.
How many areas are used in this circuit is called number of slices. Number of slices will be reduced.

\section{Number of IOBs}

All input output port used in this circuit are combined called number of input output buffer switch.Number of IOBs will be reduced.

\section{Maximum Combinational Path Delay}

Maximum delay for signal propagation is called the maximum combinational path delay.Maximum combinational will be reduced.

\section{EXPECTED OUTCOME}

This research paper expects to have the following outcomes by the end of the thesis.

- An analysis of the 4-bit, 8-bit, 12-bit, 16-bit and 32bit QSD addition/ sub-traction.

- The delay and power consumption of the QSD addition/ sub-traction will be reduced by using DPG reversible gate.

- Evaluation parameters will be calculated through proposed algorithm and compared with the base paper.

\section{CONCLUSION}

Reversible logic may be a promising computing design paradigm that presents a technique for constructing computers that produce no heat dissipation. Reversible computing emerged as results of the applying of quantum physics principles towards the event of a universal computing device [1]. Specifically, the basics of reversible computing area unit supported the link between entropy, heat transfer between molecules in an exceedingly system, the chance of a quantum particle occupying a specific state at any given time, and the quantum electrodynamics between electrons once they are in close proximity. The basic principle of reversible computing is that a bijective device with an identical number of input and output lines can produce a computing setting wherever the electrodynamics of the system provide calculation of all future states based on best-known past states, and also the system reaches each attainable state, leading to no heat dissipation [2].

\section{REFERENCES}

[1] Simranjeet Singh Sudan and Manish Singhal, "MIG and COG Reversible Logic gate Based QSD Addition / Subtraction", International Conference on Computing, Communication and Automation (ICCCA2017).

[2] Radhika Thakur, Shruti Jain and Meenakshi Sood, FPGA Implementation of Unsiged Multiplier Circuit based on Quaternary Signed Digit Number System", IEEE Conference on Signal Processing, Computing and Control, IEEE 2017.

[3] Purva Agarwal and Pawan Whig, "Low Delay Based 4 Bit QSD Adder / Subtraction Number System By Reversible Logic Gate”, 2016 8th International Conference on Computational Intelligence and Communication Networks.

[4] Ameya N. Bankar, Shweta Hajare, "Design of Arithmetic Circuit Using Quaternary Signed Digit Number System", International Conference on Communication and Signal Processing, April 3-5, 2014, India. 
[5] Tanay Chattopadhyay and Tamal Sarkar, "Logical Design of Quaternary Signed Digit Conversion Circuit and its Effectuation using Operational Amplifier", Bonfring International Journal of Power Systems and Integrated Circuits, Vol. 2, No. 3, December 2012.

[6] Krishna Murthy, Gayatri G, Manoj Kumar "Design of Efficient Adder Circuits Using Proposed Parity Preserving Gate" VLSICS Vol.3, No.3, June 2012.

[7] M. D. Saiful Islam and Z. Begum, Reversible Logic Synthesis Of Fault Tolerant Carry Skip BCD Adder, Journal of Bangladesh Academy of Sciences, Vol. 32, No. 2, 193-200, 2008.

[8] M. K. Thomsen, R. Gluck, H. B. Axelsen, 2010, Reversible arithmetic logic unit for quantum arithmetic, Journal of physics A: Mathematical and Theoretical, 43
(2010) $382002 \quad$ (10pp), $\quad$ doi: $\quad 10.1088 / 1751$ $8113 / 43 / 38 / 382002$

[9] M. Haghparast, K. Navi, 2008: A Novel Fault Tolerant Reversible Gate For Nanotechnology Based Systems, Am. J. Applied Sci., 5(5), 519-523.

[10] B. Parhami, Fault Tolerant Reversible Circuits, Proc. Asilomar Conf. Signals, Systems, and Computers, Pacific Grove, CA,Oct.2006.

[11] Haghparast, M. and K. Navi, " A novel fault tolerant reversible gate for nanotechnology based systems". Am. J. Appl. Sci., 5(5).2008.

[12] Jayashree H V and Ashwin S, "Berger Check and Fault Tolerant Reversible Arithmetic Component Design", 978-1- 4799 - 8364-3/ 15/\$31.00 @ 2015 IEEE 